\title{
SEHR GEEHRTER HERR VORSITZENDER !
}

Zu Ihrem 70. Geburtstag überbringen wir Ihnen die herzlichsten Glückwünsche des Arbeitsausschusses und der Mitglieder des Hansischen Geschichtsvereins in der Deutschen Demokratischen Republik. Wir dürfen dabei der Hoffnung Ausdruck geben, daß Sie noch lange Jahre fruchtbarer wissenschaftlicher Arbeit erleben und die Geschäfte der Arbeitsgemeinschaft führen können.

Die Hansische Arbeitsgemeinschaft blickt jetzt auf eine mehr als vierjährige erfolgreiche Tätigkeit zurück. Diese Tätigkeit erfolgte im Rahmen der Gesamtarbeit des Hansischen Geschichtsvereins und fand in den Jahresberichten des Vorstandes ihren positiven Niederschlag. Ihrer Initiative, Herr Vorsitzender, ist es zu verdanken, daß sich die Mitglieder des Hansischen Geschichtsvereins, soweit sie auf dem Territorium der Deutschen Demokratischen Republik wohnen, in einer Arbeitsgemeinschaft zusammenfanden und die vielfältigen Möglichkeiten ausschöpfen, die unser Staat der Arbeiter und Bauern der Wissenschaft bietet. Die Zahl der Mitglieder erhöhte sich in dieser Zeit erheblich; organisatorisch und finanziell hat sich die Lage der Arbeitsgemeinschaft ständig verbessert, und auch mit der Veröffentlichung wissenschaftlicher Monographien konnte inzwischen begonnen werden.

Diesen Publikationen werden weitere folgen, und die Hansische Arbeitsgemeinschaft freut sich, zu Ihrem Ehrentage einen Band hansegeschichtlicher Beiträge als Geburts. tagsgeschenk überreichen zu können, die in den letzten Jahren entstanden und vorwiegend von jüngeren Forschern verfaßt worden sind.

In ihnen spiegelt sich in erster Linie der wissenschaftliche Ertrag der Arbeitstagungen von Leipzig, Schwerin, Stendal und Berlin wider. Diese von Thnen angeregten und geleiteten Arbeitstagungen räumten vor allem dem Nachwuchs ein weites Feld für Referate und Diskussionen ein, begründeten und förderten aber zugleich auch einen engen persönlichen Kontakt zwischen den Vertretern der Hansekommunen. Sie ermöglichten überhaupt erst den Historikern und Archivaren, den Bibliothekaren und Lehrern, die im Bereich der Hanseforschung tätig sind, die wissenschaftliche Arbeit mit staatlichen Mitteln zu leisten. Das Resultat dieser Arbeit soll als Sammelband vorgelegt werden und von den Fortschritten auf diesem Gebiet der Geschichtswissenschaft in der Deutschen Demokratischen Republik Zeugnis ablegen.

Zugleich aber zeigt der Teilnehmerkreis, welche internationale Stellung die Hansische Arbeitsgemeinsehaft unter Ihrer Leitung erreicht hat. Der Arbeitsausschu $B$ und die Mitglieder sind sich einig darin, daß diese Erfolge ganz wesentlich Threm persönlichen Einsatz für die Hanseforschung und für den Hansischen Geschichtsyerein zu danken sind.

Die Hansische Arbeitagemeinschaft widmet Ihnen deshalb diese Sammlung von ,Hansischen Studien' als Zeichen der Hochachtung und der besonderen Verehrung mit den besten Wünschen für eine weitere schöpferische Arbeit.

Berlin-Niederschönhausen,

den 25. November 1959

Hansischer Geschichtsverein

Arbeitsgemeinschaft in der

Deutschen Demokratischen Republik

Dr. K. Höhnel

Stellvertreter des Vorsitzenden 
